\title{
Desempenho de híbridos de dendezeiro (Elaeis guineenses Jacq.) nas fases de pré-viveiro e viveiro
}

\author{
Sérgio José da Costa ${ }^{1}$, Eduardo Andrea Lemus Erasmo ${ }^{2}$, Joedna Silva ${ }^{1}$, Taynar Coelho \\ Oliveira $^{2}$ \\ ${ }^{1}$ Instituto Federal de Educação Ciência e Tecnologia do Tocantins, Campus de Gurupi, Tocantins, Brasil. E-mail: \\ sergiojose@ifto.edu.br, joedna.silva@ifto.edu.br \\ ${ }^{2}$ Universidade Federal do Tocantins, Campus Universitário de Gurupi, Tocantins, Brasil. E-mail: erasmolemus@uft.edu.br, \\ taynarcoelho@hotmail.com
}

Recebido: 24/01/2017; Aceito: 01/10/2018.

\section{RESUMO}

A palma do dendezeiro (Elaeis guineensis) é uma cultura que produz maior quantidade de óleo por unidade de área cultivada entre todas as outras plantas oleaginosas, com ciclo produtivo de 25 a 30 anos, e apresenta elevada capacidade de realizar o sequestro de carbono. Para a produção de mudas é necessário desenvolver cuidados para garantir plantas aptas a serem cultivadas em campo. Diante desse contexto, a presente pesquisa buscou estudar as fases inciais desta planta (pré-viveiro e viveiro) em região de fronteira agrícola no cerrado do Tocantins. Os materiais avaliados foram BRS 2301, BRS 7201, BRS 2528, BRS 2001 e BRS 2501. As variáveis avaliadas foram altura de planta, número de folhas, volume radicular, comprimento de raiz, diâmetro do caule, massa seca das folhas, massa seca do caule, massa seca da raízes, massa seca total e área foliar. Na fase de pré-viveiro, o híbrido BRS 2528 apresentou o melhor desempenho para as variáveis analisadas, enquanto que, na de viveiro, os híbridos BRS 2001 e BRS 7201 superaram os outros materiais. Dentre as variáveis avaliadas, as maiores diferenças entre os híbridos foram observadas quanto ao acúmulo de massa seca total e o comprimento radicular.

Palavras-chave: dendê, mudas, cerrado.

\section{Performance of hybrids of oil palm tenera (Elaeis guineensis Jacq.) in pre-nursery and nursery phases}

\begin{abstract}
The palm oil (Elaeis guineensis Jacq.) is a crop that produces the largest amount of oil per unit of cultivated area among all other oilseed plants, with a productive cycle of 25 to 30 years and has a high capacity to carry out carbon sequestration. For the production of seedlings, it is necessary to develop care to ensure plants that are able to be cultivated in the field. In this context, this study sought to study the initial phases of this plant (nursery and pre-nursery), in a region of agricultural frontier in the Savannah of Tocantins. The materials evaluated were BRS 2301, BRS 7201, BRS 2528, BRS 2001 and BRS 2501. Evaluated variables were plant height, number of leaves, root volume, root length, stem diameter, leaf dry mass, stem dry mass, root dry mass, total dry mass and leaf area. In the prée-nursery phase the hybrid BRS 2528 presented the best performance for the analyzed variables, while in the nursery, the BRS 2001 and BRS 7201 hybrids surpassed the other materials. Among the variables evaluated the major differences between the hybrids were observed as to the accumulation of total dry mass and root length.
\end{abstract}

Keywords: palm oil, seedlings, savanna. 


\section{Introdução}

A palma de óleo ou dendê (Elaeis guineensis Jacq.) é uma cultura perene tropical com elevado rendimento na produção de óleo, apresentando produtividade média de quatro toneladas de óleo/ha/ano e elevada capacidade de realizar o sequestro de carbono, podendo participar efetivamente do Movimento de Desenvolvimento Limpo (CDM) com a venda de créditos de carbono (Queiroz, 2012).

No Brasil, o cultivo do dendezeiro expandiu-se na última década, principalmente como matéria-prima para a produção do biodiesel. Seu cultivo tem sido difundido como alternativa de desenvolvimento socioeconômico regional, além de ser utilizado como forma de recuperação e aproveitamento de áreas degradadas da região amazônica. Segundo Müller e Alves (1997), a espécie apresenta melhor desenvolvimento em regiões tropicais, devido à influência das condições climáticas no processo produtivo.

Atualmente, os cultivos comerciais estão concentrados no Estado do Pará, que é o maior produtor nacional. A área colhida em 2014 foi de 126.559 hectares, o que representa $1,25 \%$ da participação mundial (Queiroz, 2012).

O Estado de Tocantins vem apresentando vocação econômica voltada para o agronegócio, representando uma das últimas fronteiras agrícolas do país com condições edafoclimáticas favoráveis, e pode proporcionar altos ganhos de produtividade, que indicam o potencial de cultivo de dendezeiros (Ramalho Filho et al., 2010). Essa cultura deve ser estudada para aumentar a área comercial, já que produz cerca de 10 vezes mais que a produtividade da soja, e para tanto, faz-se necessário tecnologia para produzir mudas de qualidade e conhecer o comportamento dessa espécie na formação das mudas.

Segundo Barcelos et al. (2001), a qualidade da muda está diretamente relacionada com a precocidade e potencial produtivo do dendê, sendo que o processo de produção de mudas consiste em duas fases, a primeira de pré-viveiro e a segunda, de viveiro.

A fase de pré-viveiro representa a formação inicial da muda, sendo realizada em área com cobertura de sombrite a $50 \%$, onde as plantas permanecem por um período de quatro meses. Esta etapa não pode se estender por mais tempo para não prejudicar a formação da muda final. Por sua vez, a fase de viveiro, realizada em área aberta, onde as mudas devem ficar por um período de 12 meses, é considerada de extrema importância, uma vez que mudas bem cuidadas têm maiores condições de sobreviver no campo e são mais resistentes em comparação àquelas mal conduzidas (Akpo et al., 2014a; Barcelos et al. 2001; Teixeira et al., 2009).

Diante da importância da formação das mudas de dendê com alta qualidade para ampliação da área comercial da espécie no país, objetivou-se com este trabalhado avaliar o desempenho de cinco híbridos de dendê nas fases de pré-viveiro e viveiro, sob condições de Cerrado no Sul do Tocantins.

\section{Material e Métodos}

O trabalho foi conduzido na Estação Experimental de Pesquisa do Campus Universitário de Gurupi, pertencente à Universidade Federal do Tocantins - UFT, localizado na latitude $11^{\circ} 43^{\prime} \mathrm{S}$ e longitude $49^{\circ} 04^{\prime} \mathrm{W}$, em altitude média de $287 \mathrm{~m}$.

Para realização do estudo utilizou-se sementes de cinco híbridos de dendezeiro (BRS 2501, BRS 2001, BRS 2301, BRS 2528 e BRS 7201) fornecidas pela Empresa Brasileira de Pesquisa Agropecuária Embrapa Amazônia Ocidental.

O delineamento experimental foi em blocos ao acaso, com cinco tratamentos (cultivares híbridos) e quatro repetições. As principais propriedades químicas e físicas do solo na camada de $0-20 \mathrm{~cm}$ de profundidade são mostradas na tabela 1 .

As mudas foram avaliadas em duas fases (préviveiro e viveiro). Na fase de pré-viveiro, as sementes pré-germinadas foram plantadas (julho de 2013) em sacos de polietileno com dimensões de $10 \times 15 \mathrm{~cm}$, seguindo a recomendação de Teixeira et al. (2009). Em seguida, as mudas foram acondicionadas em pré-viveiro com sombrite, cuja interceptação foi de $50 \%$ de luminosidade.

Quanto à adubação, a partir do terceiro mês fez-se uma solução de $400 \mathrm{~g}$ de ureia, $400 \mathrm{~g}$ de superfosfato triplo, $100 \mathrm{~g}$ de $\mathrm{KCl}$ e $100 \mathrm{~g}$ de sulfato de magnésio, dissolvidos em $100 \mathrm{~L}$ de água e aplicados em todos os tratamentos, quinzenalmente. Logo após a aplicação da solução, realizou-se uma irrigação visando eliminar o excesso do produto nas folhas da cultura. A irrigação diária foi realizada aplicando-se uma lâmina de 3,5 $\mathrm{mm} / \mathrm{dia}$, seguindo recomendações sugeridas por Rodrigues et al. (2002).

Tabela 1. Atributos químicos e físicos do solo na camada de $0-20 \mathrm{~cm}$ de profundidade. Gurupi - TO.

\begin{tabular}{cccccccc}
\hline $\begin{array}{c}\mathrm{pH} \\
\mathrm{CaCl}_{2}\end{array}$ & $\begin{array}{c}\mathrm{Ca} \\
\mathrm{cmol}_{\mathrm{c}} \mathrm{dm}^{-3}\end{array}$ & $\begin{array}{c}\mathrm{Mg} \\
\mathrm{cmol}_{\mathrm{c}} \mathrm{dm}^{-3}\end{array}$ & $\begin{array}{c}\mathrm{Al} \\
\mathrm{cmol}_{\mathrm{c}} \mathrm{dm}^{-3}\end{array}$ & $\begin{array}{c}\mathrm{P} \\
\left(\mathrm{mg} \mathrm{dm}^{-3}\right)\end{array}$ & $\begin{array}{c}\text { Areia } \\
(\%)\end{array}$ & $\begin{array}{c}\text { Silte } \\
(\%)\end{array}$ & $\begin{array}{c}\text { Argila } \\
(\%)\end{array}$ \\
\hline 5,1 & 2,2 & 1,1 & 0 & 4,6 & 79,0 & 3,8 \\
\hline
\end{tabular}


Ao final do período de pré-viveiro (área coberta com tela de sombreamento de $50 \%$ ), foi realizada uma seleção das mudas sadias, livres de anomalias, pragas e doenças, sendo que, em novembro de 2013, com 120 DAE (dias após a emergência), as mesmas foram transplantadas para a fase de viveiro (área a sol pleno) em sacos plásticos de dimensões 40 x $40 \mathrm{~cm} \mathrm{e}$ distribuídas de forma a evitar o sombreamento.

As avaliações foram realizadas no final da fase de pré-viveiro (120 dias após a emergência) e no final da fase do viveiro (mudas com 345 dias após a emergência), sendo observadas as seguintes características: i) altura de plantas $(\mathrm{cm})$ : por meio de régua, avaliou-se a altura total da planta; ii) úmero de folhas: contagem total das folhas; iii) volume de raiz: o volume de raiz foi obtido através do deslocamento de volume de água após a imersão das raízes em recipiente com volume conhecido; iv) comprimento de raiz (cm): por meio de régua, avaliou-se o comprimento da raiz; v) diâmetro do coleto $(\mathrm{cm})$ : por meio do paquímetro; vi) area foliar: a área foliar foi medida utilizando o equipamento da Licor Area Meter, modelo LI 3100; vii) massa seca foliar ( $\mathrm{g}$ ), massa seca do caule (g), massa seca da raiz $(\mathrm{g})$ e massa seca total $(\mathrm{g})$ : o material vegetal foi separado em seus componentes (folha, coleto e raízes), lavado em água corrente e acondicionado em sacos de papel, sendo colocado em estufa de circulação forçada a $70{ }^{\circ} \mathrm{C}$ por 72 horas para posterior pesagem.

As condições climáticas do período de desenvolvimento do trabalho estão representadas na Figura 1. A temperatura média no período do experimento foi de $26,2{ }^{\circ} \mathrm{C}$, estando a umidade relativa do ar em torno de $72,8 \%$, e a precipitação acumulada no período de $1.677,4 \mathrm{~mm}$.

As médias dos caracteres avaliados obtidos em cada tratamento foram comparadas pelo teste de Scott-Knott a 5\% de significância, utilizando o programa SISVAR versão 5.6 (Ferreira, 2014).

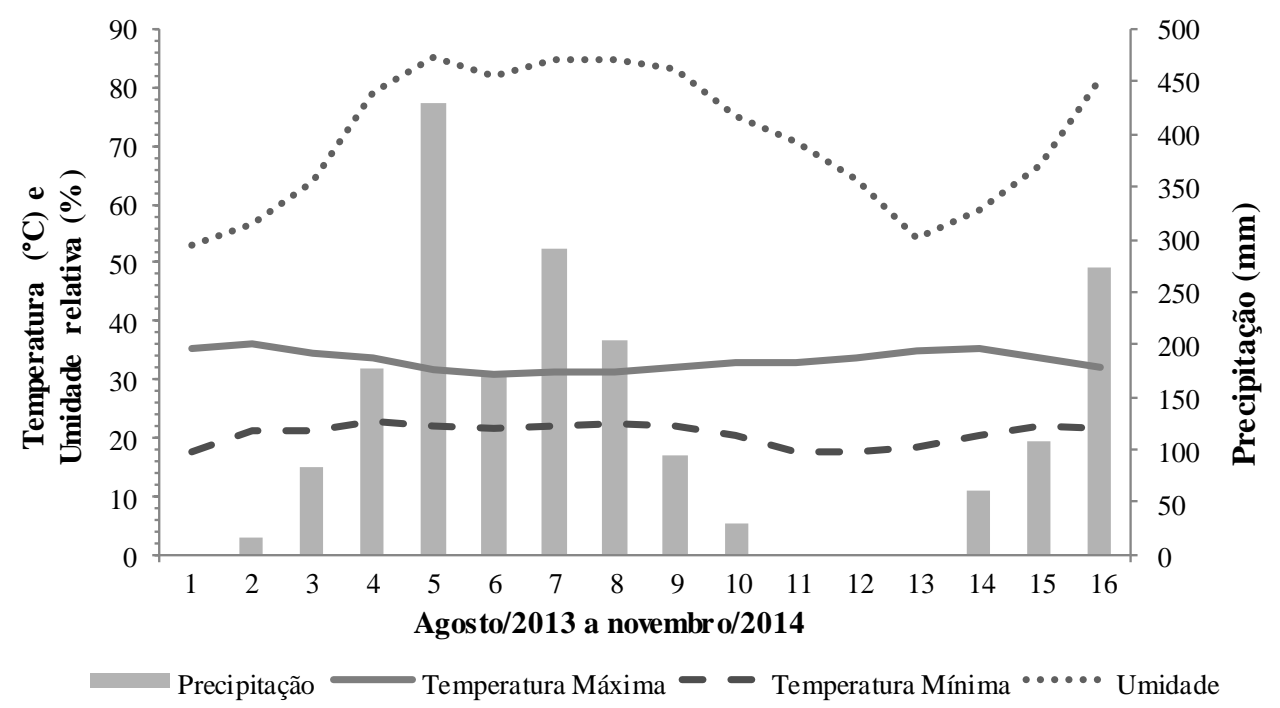

Figura 1. Dados climáticos do período experimental (agosto de 2013 a novembro de 2014). Gurupi, TO. (Estação ClimatológicaUFT, 2016).

\section{Resultados e Discussão}

Os resultados referentes à altura de planta, número de folhas, volume de raiz, comprimento de raiz e diâmetro do coleto durante a fase de pré-viveiro do dendezeiro são mostrados na Tabela 2. Evidencia-se que houve diferenças significativas entre os híbridos para as variáveis altura de planta, volume de raiz, comprimento de raiz e diâmetro do coleto. Somente não apresentou diferenças o número de folhas.

Analisando os híbridos estudados, verificou-se superioridade da altura de planta do híbrido BRS 2528, com 77,00 cm, sendo observadas as menores alturas nos híbridos BRS 2501 e BRS 7201, com 53,5 e $52 \mathrm{~cm}$, respectivamente. Assim como ocorreu com a altura de planta, o híbrido BRS 2528 apresentou maior volume de raiz $\left(28,75 \mathrm{~cm}^{3}\right)$ e diâmetro de coleto $(2,5 \mathrm{~cm})$, diferindo estatisticamente dos demais materiais (Tabela 2). Este híbrido (BRS 2528) é resultado de um programa de melhoramento executado pela Embrapa Amazônia Ocidental, e sua principal característica é o crescimento da planta (Krug et al., 2013), o que se confirma nos dados aqui obtidos e, futuramente, poderá ser um material que apresenta boa produtividade. Já no que se refere à variável número de folhas, não ocorreu diferença significativa entre os híbridos de dendezeiro, apresentando média geral 8,6 folhas (Tabela 2).

Com relação ao comprimento de raiz (Figura 2), o maior comprimento foi encontrado no híbrido BRS $2501(45,50 \mathrm{~cm})$. Esse resultado corrobora com os obtidos por Jourdan e Rey (1997), que observaram que 
o tamanho de raízes de dendê não ultrapassou $50 \mathrm{~cm}$ de comprimento na fase pré-viveiro. Teixeira et al. (2009) testando diferentes densidades de mudas de dendê, híbridos de Tenera, em tubetes na fase de pré-viveiro, obtiveram respostas semelhantes a estes testados, principalmente para a variável altura de plantas, confirmando os resultados obtidos neste estudo.



Figura 2. Raízes das mudas de dendezeiro (Elaeis guineensis Jacq.) em fase final de pré-viveiro.

Os resultados da massa seca das folhas, do caule, das raízes, massa seca total e área foliar são mostrados na Tabela 3. O híbrido BRS 2528 apresentou os maiores resultados para MSF, MSC, MSR e MST, embora a massa seca do coleto não tenha diferido do híbrido BRS 2001.
Quanto à área foliar, evidencia-se que o híbrido BRS 2001 se destacou em relação aos demais, com 882,75 $\mathrm{cm}^{2}$ aos 120 dias, possuindo oito folhas (Tabela 2), fato que provavelmente pode ser explicado pelo tamanho das folhas.

$\mathrm{Na}$ Tabela 4, descrevem-se as variáveis avaliadas em cinco híbridos de dendê na fase final de viveiro (mudas com 485 dias). Para o número de folhas não foi observada diferença significativa entre os híbridos de dendê, com média de 16,05 folhas, mas houve para a altura de planta, o volume radicular, o comprimento de raiz e o diâmetro do coleto. Ao analisar o aumento de altura dos materiais na fase de viveiro, observaram-se ganhos de $42,57 \mathrm{~cm}$ para o BRS $7201 ; 40,05 \mathrm{~cm}$ para o BRS 2001; 34,94 cm para o BRS 2301; $35,74 \mathrm{~cm}$ para o BRS 2501 e 12,83 cm para o BRS 2528 em relação à fase anterior de pré-viveiro.

O maior volume radicular foi verificado no híbrido BRS $7201(85,25 \mathrm{~cm})$, diferindo significativamente dos híbridos BRS 2001, BRS 2301, BRS 2528 e BRS 2501 $\left(54,50 \mathrm{~cm}^{3}\right)$. Jourdan e Rey (1997), trabalhando com arquitetura e desenvolvimento do sistema radicular do dendezeiro, verificaram vários tipos de formação de raízes laterais, o que pode explicar o porquê de o BRS 7201 ter sido o híbrido que apresentou menor comprimento de raiz $(43,00 \mathrm{~cm})$; entretanto, foi $\mathrm{o}$ material significativamente de maior volume de raiz $\left(85,25 \mathrm{~cm}^{3}\right)$. Para o diâmetro de coleto, os híbridos BRS 7201 e BRS 2301 apresentaram os maiores valores, com 89,25 e $89,00 \mathrm{~cm}$, respectivamente.

Tabela 2. Média das variáveis biométricas de dendê em fase de pré-viveiro (aos 120 dias após a emergência). Gurupi - TO, 2016.

\begin{tabular}{|c|c|c|c|c|c|c|c|c|c|c|}
\hline \multirow{2}{*}{$\begin{array}{l}\text { Cultivar } \\
\text { BRS } 2528\end{array}$} & \multicolumn{2}{|c|}{$\begin{array}{l}\text { Altura de planta } \\
(\mathrm{cm})\end{array}$} & \multicolumn{2}{|c|}{$\mathrm{N}^{\circ}$ de folhas } & \multicolumn{2}{|c|}{$\begin{array}{l}\text { Volume radicular } \\
\left(\mathrm{cm}^{3}\right)\end{array}$} & \multicolumn{2}{|c|}{$\begin{array}{l}\text { Comprimento de raízes } \\
(\mathrm{cm})\end{array}$} & \multicolumn{2}{|c|}{$\begin{array}{l}\text { Diâmetro de caule } \\
(\mathrm{cm})\end{array}$} \\
\hline & 77,00 & $\mathrm{a}$ & 8,50 & $\mathrm{a}$ & 28,75 & $\bar{a}$ & 36,00 & $\mathrm{~d}$ & 2,50 & $\bar{a}$ \\
\hline BRS 2301 & 59,00 & $\mathrm{~b}$ & 9,25 & $\mathrm{a}$ & 21,75 & $\mathrm{~b}$ & 42,00 & $\mathrm{~b}$ & 2,00 & $\mathrm{~b}$ \\
\hline BRS 2001 & 56,50 & $\mathrm{~b}$ & 8,50 & $\mathrm{a}$ & 18,25 & $\mathrm{c}$ & 38,75 & $\mathrm{c}$ & 2,00 & $\mathrm{~b}$ \\
\hline BRS 7201 & 52,00 & $\mathrm{c}$ & 8,25 & $\mathrm{a}$ & 20,75 & $\mathrm{c}$ & 36,75 & $\mathrm{~d}$ & 2,00 & $\mathrm{~b}$ \\
\hline BRS 2501 & 53,50 & $\mathrm{c}$ & 8,50 & $\mathrm{a}$ & 17,50 & $\mathrm{c}$ & 45,50 & $\mathrm{a}$ & 2,00 & $\mathrm{~b}$ \\
\hline Média & 59,60 & & 8,60 & & 21,40 & & 39,80 & & 2,10 & \\
\hline $\mathrm{CV}(\%)$ & 3,47 & & 8,75 & & 3,82 & & 3,21 & & 12,30 & \\
\hline
\end{tabular}

Médias seguidas de mesma letra minúscula na coluna, para cada variável, não diferem entre si a $5 \%$ de probabilidade pelo Scott-Knott.

Tabela 3. Médias da massa seca dos componentes vegetativos de híbridos de dendezeiro e área foliar em pré-viveiro (aos 120 dias após a emergência). Gurupi - TO, 2016.

\begin{tabular}{|c|c|c|c|c|c|c|c|c|c|c|}
\hline \multirow{2}{*}{$\begin{array}{l}\text { Cultivar } \\
\text { BRS } 2528\end{array}$} & \multicolumn{2}{|c|}{$\operatorname{MSF}(\mathrm{g})$} & \multicolumn{2}{|c|}{$\operatorname{MSC}(\mathrm{g})$} & \multicolumn{2}{|c|}{$\operatorname{MSR}(\mathrm{g})$} & \multicolumn{2}{|c|}{$\operatorname{MST}(\mathrm{g})$} & \multicolumn{2}{|c|}{ Área foliar $\left(\mathrm{cm}^{2}\right)$} \\
\hline & 7,02 & $\mathrm{a}$ & 5,25 & $\mathrm{a}$ & 4,00 & $\mathrm{~A}$ & 16,62 & $\mathrm{a}$ & 848,25 & $\mathrm{~b}$ \\
\hline BRS 2001 & 5,50 & $\mathrm{~b}$ & 4,75 & $\mathrm{a}$ & 3,00 & $\mathrm{~B}$ & 13,09 & $\mathrm{~b}$ & 882,75 & $\mathrm{a}$ \\
\hline BRS 2301 & 5,55 & $\mathrm{~b}$ & 4,00 & $\mathrm{~b}$ & 3,00 & $\mathrm{~B}$ & 12,38 & $\mathrm{~b}$ & 820,66 & $\mathrm{~b}$ \\
\hline BRS 7201 & 5,78 & $\mathrm{~b}$ & 3,50 & $\mathrm{~b}$ & 2,75 & $\mathrm{~b}$ & 11,87 & $\mathrm{~b}$ & 734,50 & $\mathrm{c}$ \\
\hline BRS 2501 & 4,74 & $\mathrm{c}$ & 2,75 & $\mathrm{c}$ & 2,25 & $\mathrm{c}$ & 9,62 & $\mathrm{c}$ & 613,07 & $\mathrm{~d}$ \\
\hline Média & 5,71 & & 4,05 & & 3,00 & & 12,71 & & 779,84 & \\
\hline $\mathrm{CV}(\%)$ & 8,82 & & 11,49 & & 10,54 & & 6,02 & & 2,88 & \\
\hline
\end{tabular}

MSF - Massa seca de folhas; MSC - Massa seca do caule; MSR - Massa seca da raiz; MST - Massa seca total; Área Foliar. Médias seguidas de mesma letra minúscula na coluna, para cada variável, não diferem entre si a $5 \%$ de probabilidade pelo Scott-Knott. 
Tabela 4. Médias de altura, número de folhas, volume de raízes, comprimento de raízes e diâmetro de coleto de híbridos de dendezeiro na Fase de Viveiro (plantas com 345 dias após a emergência). Gurupi - TO, 2016.

\begin{tabular}{|c|c|c|c|c|c|c|c|c|c|c|}
\hline \multirow{2}{*}{$\frac{\text { Cultivar }}{\text { BRS } 2001}$} & \multicolumn{2}{|l|}{$\begin{array}{l}\text { Altura } \\
(\mathrm{cm})\end{array}$} & \multicolumn{2}{|c|}{$\mathrm{N}^{\circ}$ de folhas } & \multicolumn{2}{|c|}{$\begin{array}{l}\text { Volume radicular } \\
\left(\mathrm{cm}^{3}\right)\end{array}$} & \multicolumn{2}{|c|}{$\begin{array}{l}\text { Comprimento de raiz } \\
(\mathrm{cm})\end{array}$} & \multicolumn{2}{|c|}{$\begin{array}{l}\text { Diâmetro do caule } \\
(\mathrm{cm})\end{array}$} \\
\hline & 118,00 & $\mathrm{a}$ & 15,75 & $\mathrm{a}$ & 64,00 & $\mathrm{~b}$ & 48,00 & $\mathrm{~b}$ & 80,00 & $\mathrm{~b}$ \\
\hline BRS 7201 & 108,00 & $\mathrm{~b}$ & 16,00 & $\mathrm{a}$ & 85,25 & $\mathrm{a}$ & 43,00 & $\mathrm{c}$ & 89,00 & $\mathrm{a}$ \\
\hline BRS 2301 & 104,00 & $\mathrm{~b}$ & 15,75 & $\mathrm{a}$ & 64,50 & $\mathrm{~b}$ & 51,50 & $\mathrm{a}$ & 89,25 & $\mathrm{a}$ \\
\hline BRS 2528 & 101,25 & $\mathrm{~b}$ & 15,75 & $\mathrm{a}$ & 65,75 & $\mathrm{~b}$ & 42,00 & $\mathrm{c}$ & 83,00 & $\mathrm{~b}$ \\
\hline BRS 2501 & 104,00 & $\mathrm{~b}$ & 16,50 & $\mathrm{a}$ & 54,50 & $\mathrm{c}$ & 53,25 & $\mathrm{a}$ & 84,75 & $\mathrm{~b}$ \\
\hline Média & 108,05 & & 16,05 & & 66,71 & & 47,55 & & 85,20 & \\
\hline CV (\%) & 4,87 & & 9,88 & & 6,64 & & 5,03 & & 3,66 & \\
\hline
\end{tabular}

Médias seguidas de mesma letra minúscula na coluna, para cada variável, não diferem entre si a 5\% de probabilidade pelo Scott-Knott.

Tabela 5. Médias da massa seca dos componentes vegetativos de híbridos de dendê e área foliar em fase de viveiro (345 dias após a emergência). Gurupi - TO, 2016.

\begin{tabular}{|c|c|c|c|c|c|c|c|c|c|c|}
\hline \multirow{2}{*}{$\begin{array}{l}\text { Cultivar } \\
\text { BRS } 7201\end{array}$} & \multicolumn{2}{|c|}{$\operatorname{MSF}(\mathrm{g})$} & \multicolumn{2}{|c|}{$\operatorname{MSC}(\mathrm{g})$} & \multicolumn{2}{|c|}{$\operatorname{MSR}(\mathrm{g})$} & \multicolumn{2}{|c|}{$\operatorname{MST}(\mathrm{g})$} & \multicolumn{2}{|c|}{ Área foliar $\left(\mathrm{cm}^{2}\right)$} \\
\hline & 218,75 & $\mathrm{a}$ & 235,50 & $\mathrm{a}$ & 129,25 & $\mathrm{a}$ & 576,00 & $\mathrm{a}$ & $13.276,50$ & $\mathrm{a}$ \\
\hline BRS 2301 & 217,75 & $\mathrm{a}$ & 234,25 & $\mathrm{a}$ & 75,00 & $\mathrm{c}$ & 512,25 & $\mathrm{~b}$ & $11.527,00$ & $\mathrm{~b}$ \\
\hline BRS 2001 & 195,75 & b & 178,00 & $\mathrm{~b}$ & 108,00 & $\mathrm{~b}$ & 464,50 & b & $12.344,75$ & $\mathrm{a}$ \\
\hline BRS 2528 & 144,25 & $\mathrm{c}$ & 220,25 & $\mathrm{a}$ & 122,75 & $\mathrm{a}$ & 489,50 & $\mathrm{~b}$ & $11.329,75$ & $\mathrm{~b}$ \\
\hline BRS 2501 & 146,25 & $\mathrm{c}$ & 178,00 & $\mathrm{~b}$ & 69,50 & $\mathrm{c}$ & 386,00 & $\mathrm{c}$ & $10.137,50$ & $\mathrm{~b}$ \\
\hline Média & 184,55 & & 207,70 & & 100,90 & & 485,65 & & $117.23,00$ & \\
\hline CV $(\%)$ & 5,98 & & 7,70 & & 9,38 & & 9,56 & & 9,18 & \\
\hline
\end{tabular}

MSF - Massa seca de folhas; MSC - Massa seca do caule; MSR - Massa seca das raízes; MST - Massa seca total. Médias seguidas de mesma letra minúscula na coluna não diferem entre si a $5 \%$ de probabilidade pelo Scott-Knott.

O mesmo não aconteceu com a variável comprimento de raiz, onde os híbridos BRS 2501 e BRS 2301 foram os materiais com maior desenvolvimento radicular, com 53,25 e $51,50 \mathrm{~cm}$, respectivamente, seguido do híbrido BRS $2001(48,00 \mathrm{~cm})$ e BRS 7201 (43,00 cm). O BRS 2528 foi o híbrido com menor desenvolvimento radicular $(42,00 \mathrm{~cm})$ entre todos os materiais estudados.

Quanto ao acúmulo de massa seca (Tabela 5), o BRS 7201 apresentou superioridade em relação aos demais híbridos. Quando se faz uma análise dos resultados da massa seca foliar, observa-se que houve uma diferença estatística de 46,63 e 41,05\%, respectivamente, dos materiais BRS 2501 e BRS 2528 em comparação com o BRS 7201, que obteve melhores resultados para esta variável.

Para MSC foram observados menores valores para os cultivares BRS 2501 e BRS 2001 (32,22 e 41,96\%, respectivamente) em comparação ao BRS 2301. Para a variável MSR, os híbridos BRS 7201 e BRS 2528 obtiveram rendimento superior, diferindo estatisticamente dos demais. Cabe destacar que a maior variação observada entre os materiais quanto ao acúmulo de massa seca foi para o parâmetro massa seca de raiz. Akapo et al. (2014b) destacaram a importância do monitoramento das mudas nestas fases estudadas, por garantir a produção de plantas bem desenvolvidas e aptas a serem plantadas em campo. Bovi et al. (1993), estudando a pupunha (Bactris gasipaes), relacionam que o diâmetro do coleto e o número de folhas podem ser importantes parâmetros a serem avaliados durante o processo de formação de mudas de palmáceas e estão diretamente relacionados também com o desenvolvimento dessas plantas no campo.

Já Gonçalves e Mello (2000) atribuíram para a maioria das espécies vegetais superiores grande importância à variável comprimento das raízes, considerando este como um dos melhores parâmetros, pois está diretamente ligado à absorção de água e nutrientes pelas plantas. Tendo em vista o exposto, infere-se que o híbrido BRS 7201 pode ser o material com melhor capacidade de desenvolvimento nas fases seguintes.

\section{Conclusões}

$\mathrm{Na}$ fase de pré-viveiro, o híbrido BRS 2528 apresentou os maiores valores nas variáveis avaliadas.

$\mathrm{Na}$ fase viveiro, os híbridos BRS 2001 e BRS 7201 superaram os demais nas variáveis avaliadas, exceto para comprimento de raiz.

As maiores diferenças entre os híbridos corresponderam ao acúmulo de massa seca total e comprimento radicular.

\section{Referências Bibliográficas}

Akpo, E., Stomph, TJ., Kossou, D.K., Struik, P.C. 2014a. Growth dynamics of tree nursery seedlings: The case of oil palm. Scientia Horticultura, 175, 251-257. 
Akpo, E., Stomph, T.J., Kossou, D.K., Omore, A.O., Struik, P.C. 2014b. Effects of nursery management practices on morphological quality attributes of tree seedlings at planting: The case of oil palm (Elaeis guineensis Jacq.). Forest Ecology and Management, 324, 28-36.

Barcelos, E., Rodrigues, M.R.L., Santos, J.A., Cunha, R.N.V. 2001. Produção de Mudas de Dendezeiro na Amazônia. Manaus: Embrapa Amazônia Ocidental. 11p. (Circular Técnica 8).

Bovi, M.L.A. 1993. Palmito pupunha: Informações básicas para o cultivo. In: Encontro Sobre Produção de Palmito. Piracicaba, CALQ, 12-23.

Ferreira, D.F. 2014. Sisvar: a Guide for its Bootstrap procedures in multiple comparisons. Ciência Agrotecnologia, $38,109-112$.

Gonçalves, J.L.M., Mello, S.L.M. 2000. O sistema radicular das árvores. In: Gonçalves, J. L. M.; Benedetti, V. (Ed.). Nutrição e fertilização florestal. Piracicaba: IPEF, 309-350.

Jourdan, C., Rey, H. 1997. Architecture and development of the oil-palm (Elaeis guineensis Jacq.) root system. Plant and Soil, 189, 33-48.

Krug, C., Bittencourt, D.M., Barcelos, E.C., Rodrigues, M.R.L., Angelo, P.C.S., Rocha, R.N.C., Cunha, R.N.V.,
Quisen, R.C., Lopes, R.L., Rios, S.A., Lima, W.A.A. 2013. Plano estratégico da Embrapa Amazônia Ocidental para a cultura do dendezeiro. Manaus: Embrapa Amazônia Ocidental. 73 p. (Documento 102).

Müller, A.A., Alves R.M. 1997. A dendeicultura na Amazônia brasileira. Belém: Embrapa.Amazônia Oriental. 44p. (Documento 91).

Queiroz, A.G., França L., Ponte, M.X. 2012. The life cycle assessment of biodiesel from palm oil ("dende") in the Amazon. Biomass and Bioenergy, 36, 50-59.

Ramalho Filho A., Motta P.E. 2010. Zoneamento Agroecológico do Dendezeiro para as áreas desmatadas da Amazônia Legal. Rio de Janeiro, Brasil: Embrapa Solos.

Rodrigues, M.R.L., Amblard, P., Barcelos, E., Macedo, J.L., Cunha, R.N.V., Tavares, A.M. 2002. Avaliação do estado nutricional do dendezeiro: análise foliar. Manaus: Embrapa Amazônia Ocidental, 9p. (Circular Técnica 11).

Teixeira, P.C., Rodrigues, H.S., Lima, W.A.A., Rocha, R.N.C., Cunha, R.N.V., Lopes, R. 2009. Influência da disposição dos tubetes e da aplicação de fertilizantes de liberação lenta, durante o pré-viveiro, no crescimento de mudas de dendezeiro (Elaeis guineensis Jacq.) Revista Ciência Florestal, 19, 157-168. 